\title{
Cardiac Vagal Tone, defensiveness, and motivational style
}

\author{
Hallam L. Movius, John J.B. Allen* \\ Department of Psychology, Cognitive Science and Neuroscience, University of Arizona, \\ P.O. Box 210068, Tucson, AZ 85721-0068, USA
}

Received 27 March 2002; accepted 30 March 2004

Available online 28 May 2004

\begin{abstract}
Cardiac Vagal Tone has been proposed as a stable biological marker for the ability to sustain attention and regulate emotion [Porges, Monographs of the Society for Research in Child Development 59 (1994) 167-186]. Vagal tone is a physiological index of parasympathetic nervous system influence on the heart that has predicted a number of emotional behaviors and styles in infants, children, and adults. Little research, however, has sought to explore the link between vagal tone and established variables relating to personality and self-regulation. In this study, vagal tone was collected during 5-min baseline, stress, and recovery periods. Subjects $(n=98)$ also completed a short form of the Marlowe-Crowne Social Desirability Scale, the Behavioral Activation and Behavioral Inhibition Scales, the Taylor Manifest Anxiety Scale, the Self-Consciousness Scale, and the Openness to Experience subscale of the Five Factor Personality Inventory. Poorer modulation for vagal tone was associated with greater social anxiety, while lower vagal tone across recording periods was associated with greater defensiveness and lower behavioral activation sensitivity.
\end{abstract}

(C) 2004 Elsevier B.V. All rights reserved.

Keywords: Vagal tone; RSA; Defensiveness; Marlowe-Crowne

\section{Cardiac Vagal Tone, defensiveness, and motivational style}

A growing body of psychophysiological research has focused on identifying autonomic correlates of temperament and health vulnerabilities. Cardiac Vagal Tone has received increasing attention, as an index of the extent to which the vagus nerve mediates

* Corresponding author. Tel.: +1-520-621-7447; fax: +1-520-621-9306.

E-mail address: jallen@u.arizona.edu (J.J.B. Allen). 
parasympathetic influence on the heart and other key organs (Porges, 1995). Accumulating evidence suggests that vagal tone may represent an important underlying mechanism in emotional, self-regulatory, and behavioral processes (Porges, 1991, 1995; Eisenberg et al., 1995; Gottman et al., 1996) as well as psychopathology (Beauchaine, 2001).

Few studies to date have examined the relationship between vagal tone and motivationrelated variables in the adult personality literature, such as defensiveness (Weinberger, 1990; Lane et al., 1990), openness to experience (Costa and McCrae, 1985, 1992), and approach-avoidance tendencies (Davidson, 1998). In recent years, measures of motivational tendency have been found to relate to specific patterns of frontal brain activity (Coan and Allen, 2003, 2004; Davidson, 1998; Harmon-Jones and Allen, 1997, 1998) that may underlie both personality tendencies and vulnerabilities to depression and anxiety (Fowles, 1987, 1988, 1994; Harmon-Jones and Allen, 1997). Moreover, while significant literatures exist on all of these variables in the social, physiological, clinical, and personality domains, few if any studies have examined relationships between these variables in a single sample of adults.

\subsection{Cardiac Vagal Tone}

The vagus nerve has two distinct source nuclei which are thought to support two related but distinct regulatory purposes. One path originates in the lateralized dorsal motor nucleus (DMX) and is believed to mediate the process of orienting to a stimulus or the environment by helping to decelerate heart rate and through influences on the so-called "vegetative" lower organs (see Beauchaine, 2001; Porges, 1995 for reviews). A second vagal pathway originates in the nucleus ambiguus (NA) and terminates on those organs associated with emotion and communication (heart, soft palate, pharynx, larynx, esophagus, bronchi, facial muscles). Under resting conditions the NA path of the vagus acts as a kind of "brake," firing a rapid and continuous signal (or "tonus") to the sino-atrial node of the heart; without such influence the heart would race along at over 100 beats per minute. Tonic vagal influence (often referred to as "vagal tone") has been correlated to a variety of attentional and self-regulatory processes in infants, children, and adults.

While this tonic influence is adaptive at many moments, the capacity to temporarily suppress vagal influence-i.e., phasic regulation — appears to mediate attentional and emotional processes that allow an organism to optimally engage or cope with environment challenges (Beauchaine, 2001; Gottman et al., 1996; Porges, 1991, 1995; Richards, 1985). Resting vagal influence and the capacity to suppress this influence have been found to be strongly related (e.g., $r=.64$; Katz and Gottman, 1997), but the precise distinction between these mechanisms and their concomitant behavioral processes is not yet clearly understood.

\subsubsection{Measuring vagal influence}

Vagal input is rhythmically interrupted with each respiratory cycle. During inspiration, heart rate momentarily increases as vagal influence is momentarily suppressed; during expiration it decreases as vagal influence resumes. Although heart rate can vary from moment to moment for reasons other than vagal influence (e.g., in response to thermoregulatory changes, physical movement, changes in blood pressure), respiration is reliably periodic. The strength of this tonic vagal influence can be assessed by examining heart rate variability 
that is tied to the respiratory cycle; such variation is called respiratory sinus arrhythmia (RSA; Porges et al., 1973, 1994). RSA has been widely used as an index of vagal tone (Porges et al., 1994; Berntson et al., 1993; Berntson et al., 1997). In assessing vagal tone, investigators have measured both resting RSA during a baseline period (e.g., Porges et al., 1994) and the modulation of RSA in response to task demands (e.g., Porges et al., 1996; Hughes and Stoney, 2000). The latter is determined by examining the difference between the RSA level at baseline and RSA during the task of interest.

\subsection{Vagal tone and personality}

A host of findings suggest that human subjects with greater vagal influence may be dispositionally more open to experience and better able to self-regulate (Porges et al., 1994; Porges, 1995). In infants, children, and adults, higher vagal tone is associated with greater reactivity to stimuli, greater ability to maintain attention on stimuli, and greater ability to self-soothe under duress. Higher vagal tone is associated in infancy with greater heart-rate acceleration and behavioral reactivity to painful stimuli (e.g., having blood drawn), greater interest and attention toward novel stimuli, and less distractibility (see Porges et al., 1994 for a review). Evidence also suggests that higher vagal tone is associated with greater facial expression of emotion and heightened interest and joy expressions (Field et al., 1982; Stifter et al., 1989).

Similar results have been found with both child and adult subjects. In children, higher vagal tone has been found to correlate with greater emotional expression (Cole et al., 1996) and fewer symptoms of psychopathology (Pine et al., 1998; Porges et al., 1996). In one longitudinal study, Katz and Gottman (1995) found that high vagal tone appeared to serve as a buffer from the effects of marital hostility. In adults, higher vagal tone (resting RSA) has been shown to predict greater self-reported regulatory control and decreased negative emotional arousal in the face of moderate-to-high level stressors (Fabes and Eisenberg, 1997).

In clinical disordered adult populations vagal tone has also been implicated as a potentially important self-regulatory mechanism. Clinically anxious subjects exhibit lower resting vagal tone (as indexed by RSA) and less ability to suppress it (Lyonfields et al., 1995; Friedman and Thayer, 1998). In addition, inducing panic symptoms through hyperventilation or sodium lactate causes vagal influence to decrease (George et al., 1989). Several studies suggest that patients with depression show decreased heart rate variability compared with controls (Carney et al., 1995; Rechlin et al., 1994; Rechlin et al., 1995; Roose et al., 1989). Not all studies, however, find this pattern (Moser et al., 1998; Rechlin, 1994). The role of lowered vagal tone in depression may further be state-specific, as patients showing increased vagal tone across treatment exhibited a clinically significant response to treatment, whereas subjects with minimal change in vagal tone did not respond to treatment (Chambers and Allen, 2002). Moreover, vagal tone has been hypothesized to interact with depression to produce clinically significant cardiac events (Sloan and Bigger, 1991).

\subsection{Summary}

Vagal tone indexes a physiological process that varies between individuals, and that appears to predict greater willingness to approach and to respond to both internal and 
external stimuli. Little research, however, has explored the connections between vagal tone and motivation-related measures of personality. The purpose of the present study was, therefore, to test a series of hypotheses with respect to widely used personality measures that are primarily used to assess motivational and emotional styles.

\subsection{The present study-hypotheses}

\subsubsection{Anxiety}

Two self-report measures were selected to attempt to conceptually replicate previous studies on the relationship between vagal tone and anxiety, but within the nonpathological range: the Taylor Manifest Anxiety Scale (MAS; Taylor, 1953), and the Social Anxiety Subscale of the Self Consciousness Scale (Fenigstein, 1975). It was hypothesized that higher vagal tone, as indexed by higher resting RSA and greater capacity to suppress RSA during a stressful task would predict less self-reported anxiety, as assessed by both scales.

\subsubsection{Defensiveness}

The Marlowe-Crowne Social Desirability Scale (MC) was originally developed to measure socially desirable responding. It consists of a series of true-false statements about the self that provide the subject with the opportunity to deny negative but ubiquitous traits ("I sometimes feel resentful when I don't get my way") and to endorse unrealistically positive ones ("no matter who I am talking to, I am always a good listener"). A careful review of studies using the scale suggests, however, that the scale actually measures self-deception rather than an attempt to appear desirable to others; subjects who score high on the scale actually seem to believe what they are reporting about themselves and try to behave accordingly (Weinberger, 1990). Previous research has shown that a subset of defensive subjects-those repressive defensive individuals who report low anxiety—exhibit greater reactivity to threatening stimuli (Weinberger et al., 1979; Schwartz, 1990) as assessed by skin conductance and heart rate reactivity. Additionally, high defensive individuals showed generally increased heart-rate reactivity to mental and physical stressors, although this relationship was modulated by gender and cynical hostility (Shapiro et al., 1995). In high-defensive men, greater HR reactivity was seen among those low in hostility, but in low-defensive men higher HR reactivity was seen among those high in hostility Miller (1993) proposed that defensive subjects exhibited greater cardiovascular reactivity to the degree that the task stressors elicited sympathetic nervous system response.

An alternative hypothesis has yet to be examined: if defensive subjects have greater reactivity generally, it may be that they are less able to modulate parasympathetic activity in response to stimuli (Weinberger, 1990). The second hypothesis is, therefore, that subjects higher in defensiveness would exhibit lower baseline vagal tone and less capacity for suppression of it in response to a stressful task.

\subsubsection{Behavioral activation and inhibition}

Previous research has generally found a relationship between approach-related behaviors and resting vagal tone (Richards and Cameron, 1985; Fox, 1989), although Beauchaine (2001) suggests that behavioral activation and vagal tone and reactivity may be dissociable as a function of particular personality traits and psychopathology. It was hypothesized that 
in this college student sample higher vagal tone (as indexed by resting RSA) would predict higher Behavioral Activation, and lower Behavioral Inhibition, as measured by the BIS-BAS (Carver and White, 1994), a self-report inventory of approach and withdrawal-related motivational style.

\section{Method}

\subsection{Participants}

Nine hundred twenty-one undergraduates had previously completed a survey that included a measure of defensiveness, the short form of the Marlowe-Crowne Social Desirability Inventory (MC; Reynolds, 1982). Equal numbers of high- and low-defensive participants, based upon a median split, were randomly sampled to be recruited by telephone for an experiment on "personality characteristics," for which they would receive credits toward a course in introductory psychology. Participants who were currently taking cardiovascular medications or those with a history of cardiac disease were ruled ineligible for the study. No additional information regarding cardiovascular functioning (e.g., family history of hypertension) was obtained or included in the present study. A total of 114 participants were contacted and agreed to participate in the study. Fourteen of these were excluded from analyses due to electrocardiogram (ECG) recording difficulties. This left 100 participants, 48 males and 52 females, for further analysis. Two more were excluded (see below) for deviantly low resting RSA, leaving a final sample of 98 participants (47 men and 51 women). The excluded participants did not differ significantly from the included participants on any of the personality measures reported here.

\subsection{Procedures}

After signing a consent form, participants were prepared for psychophysiological recording. Three $\mathrm{Ag}-\mathrm{AgCl}$ electrodes were attached to each participant in a Lead-II formation (see Papillo and Shapiro, 1990) ${ }^{1}$ wherein one electrode is affixed to the right forearm, a second to the back of the left leg below the calf muscle, and a third (ground) to the left forearm. Impedances were reduced to less than $20 \mathrm{~K}$ ohms on all electrodes for each participant. ECG signals were amplified 1000 times with a bandpass of .05 to $100 \mathrm{~Hz}$, and then digitized at $500 \mathrm{~Hz}^{2}$.

\subsubsection{Tasks for recording}

After being prepared for electrocardiographic recording, participants were seated in a sound-dampened chamber that contained two audio speakers for communication and a video camera. The experimenter explained that the video camera was only for use in monitoring

\footnotetext{
${ }^{1}$ The first six of these 98 subjects were run using a Lead I formation (one electrode on each forearm with a ground on the left wrist), but the Lead II was then used as it produced a more consistent R-spike.

${ }^{2}$ Five subjects were inadvertently digitized at $200 \mathrm{~Hz}$. Removing them from the analysis did not change the pattern of significant findings.
} 
participants visually to make sure the experiment was progressing according to procedure. Participants were then asked to sit quietly for a period of $5 \mathrm{~min}$ so that the experimenter could collect "some baseline readings." The signal data collected during this period yielded baseline vagal tone. Participants were then asked to perform serial paced mental arithmetic (counting backward in varying intervals, starting with a four-digit number) for five roughly 1-min periods to induce attention-focusing activity (Sloan et al., 1991; Porges et al., 1975). A task that demands attentional resources should cause participants to suppress vagal tone. The change in RSA from baseline thus constituted a measure of vagal suppression. Finally, participants were lead through a scripted guided relaxation exercise for several minutes. Following the exercise, ECG was recorded for $5 \mathrm{~min}$, with the difference in RSA between the suppression and recovery periods yielding a measure of vagal recovery.

After this task, participants exited the chamber and were disconnected from the recording electrodes. Participants were then taken to a separate lab across the hall to complete a pencil-and-paper questionnaire packet.

\subsubsection{Personality questionnaires}

The questionnaire packet included the Behavioral Inhibition and Behavioral Activation Scales (BIS-BAS), the Self-Consciousness Scale (SCS), and the Taylor Manifest Anxiety Scale (MAS). The Openness to Experience subscale (OE) of the NEO-PI (Costa and McCrae, 1985, 1992; McCrae, 1996; McCrae and Costa, 1994; McCrae and John, 1992) had been completed previously during the survey at the beginning of the semester, the same survey during which the Marlowe-Crowne had been completed. Total scores and reliability coefficients were derived for the BIS-BAS, the Social Anxiety subscale of the SCS, the OE, and the MAS.

\subsubsection{Follow-up questionnaire and debriefing}

After leaving the participant alone to complete the questionnaire packet, the experimenter stayed out of sight until the participant emerged from the room. Participants were then asked to fill out a brief survey, to assess whether they had guessed the nature of the experiment (no subject had) and to ensure that subjects had experienced the paced serial arithmetic task as challenging. They were then debriefed and thanked for their participation.

\subsection{Data reduction}

\subsubsection{ECG signal reduction}

Raw digitized ECG signals were analyzed off-line. Signals were first filtered with a digital band-pass filter $(-92 \mathrm{~dB}$ per octave, $3-50 \mathrm{~Hz})$. The first derivative of the filtered waveform was then obtained, which facilitated identification of the R-spike. An interbeat interval (IBI) series was generated to a file using a peak detection algorithm, after which the series was screened by hand and corrected for artifacts. Heart period variability in the high frequency band $(.12-.4 \mathrm{~Hz}$ ) was extracted using CMET software (Allen, 2002), which produces an estimate of RSA that correlates .99 with that produced by MX Edit software (Delta-Biometrics Inc., 1988-1993). Whereas MX Edit uses a polynomial filter, the CMet uses an optimal finite impulse response digital filter using FWTGEN V3.8 from Cook and Miller (1992). CMet converts the IBI series to a time-series sampled at $10 \mathrm{~Hz}$, 
filters the series using a 241-point optimal finite impulse response filter with half-amplitude frequencies of .12 and $.40 \mathrm{~Hz}$, and then takes the natural log of the variance of the filtered waveform as the estimate of RSA. An estimate of RSA was derived for each 5-min recording period (baseline, suppression, and recovery). Upon inspection of the distribution of RSA, two subjects were identified whose RSA fell more than three standard deviations below the mean for the sample, both in the abnormally low direction. They were, therefore, excluded from further analyses (as mentioned above).

\section{Results}

Descriptive statistics for all measures are presented in Table 1. Relationships between vagal measures and other variables of interest were analyzed initially through inspection of the zero-order correlation matrix. Three measures of vagal influence were examined: RSA at baseline, RSA suppression (baseline RSA minus RSA during task), and RSA recovery (RSA during relaxation minus RSA during task). Subtractions for these latter measures were performed such that larger values were indicative of greater suppression and also greater recovery. Additionally, in order to identify individuals whose patterns of RSA differed across tasks, and to test for gender effects and interactions, repeated measures ANOVAs on RSA level during each period (Baseline, Suppress, Recovery) were employed using median splits of key variables to generate categorical independent variables. Participant's gender was included in these ANOVAs, but is only reported for the infrequent instances where significant gender effects or interactions were found. For all repeated measures analyses the Greenhouse-Geisser corrected $p$-values are reported, along with the uncorrected degrees of freedom. In cases where data were missing from a scale, total scores were not calculated; degrees of freedom may, therefore, vary from analysis to analysis.

Table 1

Means ( \pm S.D.) by gender for main measures

\begin{tabular}{lccc}
\hline & Female & Male & Total Sample \\
\hline RSA & $6.7(1.0)$ & $6.8(1.6)$ & $6.8(1.0)$ \\
Defensiveness & $5.7(3.2)$ & $5.7(2.6)$ & $5.7(2.9)$ \\
BAS $_{\text {Total }}$ & $36.4(3.9)$ & $36.0(4.5)$ & $36.2(4.2)$ \\
BAS $_{\text {Fun }}$ & $11.5(1.8)$ & $11.8(2.4)$ & $11.7(2.1)$ \\
BAS $_{\text {Reward }}$ & $16.9(1.6)$ & $16.1(1.7)$ & $16.5(1.7)$ \\
BAS $_{\text {Drive }}$ & $10.7(2.1)$ & $11.2(2.4)$ & $11.0(2.2)$ \\
BIS & $21.7(3.1)$ & $20.1(3.4)$ & $20.9(3.3)$ \\
Anxiety & $13.0(5.7)$ & $11.5(5.0)$ & $12.3(5.4)$ \\
Anxiety & $7.8(4.7)$ & $7.0(4.2)$ & $7.4(4.4)$ \\
OE & $14.8(15.3)$ & $17.7(21.5)$ & $16.2(18.5)$ \\
\hline
\end{tabular}

Note: RSA: resting RSA at baseline; defensiveness: Marlowe-Crowne score; $\mathrm{BAS}_{\text {Total }}$ : Behavioral Activation Scale; $\mathrm{BAS}_{\mathrm{Fun}}$ : fun subscale of BAS; $\mathrm{BAS}_{\text {Reward }}$ : reward subscale; $\mathrm{BAS}_{\text {Drive }}$ : drive subscale,; BIS: Behavioral Inhibition Scale; Anxietysocial: social anxiety subscale from Self-Consciousness Scale; Anxiety Manifest: Taylor Manifest Anxiety Scale; OE: Openness to Experience scale of NEO-PI-R. 


\subsection{Preliminary analyses}

\subsubsection{Reliability of measures}

Internal consistency reliability coefficients were adequate for self-report measures: OE (.89), MC (.71), BAS (.79), BIS(.76), MAS (.82), and the social anxiety subscale of the SCS (.80). The three subscales of the BAS also exhibited adequate reliabilities (reward responsiveness $=.64$, fun-seeking $=.68$, drive $=.81$ ).

\subsubsection{Manipulation check}

As a manipulation check for the arithmetic task, a repeated measures ANOVA was performed for RSA level during the three tasks (baseline, suppression, recovery). A significant task effect $(F(2,194)=7.9, P<.01)$ indicated that subjects modulated RSA, with significantly $(P<.01)$ decreased RSA during the arithmetic (suppression) task compared to both baseline and recovery phases, which did not differ significantly $(P>.40)$ from one another. Consistent with previous studies, Table 2 shows that those individuals with higher baseline RSA displayed greater suppression (change from baseline to arithmetic task) of RSA ( $r=.41, P<.001$ ), and also better recovery (change from task to recovery period) of RSA $(r=.20, P<.05)$. In subsequent ANOVAs involving task, gender, and each personality measure, the effect of task was of course significant and is, therefore, not reported repeatedly below; only effects involving the personality measure of interest and/or gender are reported.

\subsection{Main hypotheses}

\subsubsection{Anxiety}

The hypothesis that greater resting vagal tone and greater capacity to suppress it during a stressful task would predict less self-reported anxiety, as assessed by both the Manifest

Table 2

Correlations between Cardiac Vagal Tone and motivation-related personality measures

\begin{tabular}{lccc}
\hline & Baseline & Suppression & Recovery \\
\hline Vagal measures & $.41^{* *}$ & & \\
$\quad$ Suppression & $.20^{*}$ & $.82^{* *}$ & \\
Recovery & & & .08 \\
Self-report measures & -.11 & .15 & .04 \\
MC & $.20^{\$}$ & -.03 & .06 \\
BAS $_{\text {Total }}$ & .17 & -.07 & -.09 \\
$\quad$ BAS $_{\text {Fun }}$ & $.22^{*}$ & .03 & .07 \\
BAS & .06 & .01 & -.11 \\
$\quad$ BAS & .01 & $-.33^{* *}$ \\
BIS & -.01 & $-.22^{*}$ & -.09 \\
Anxiety & -.06 & .04 & .00 \\
Anxiety & .12 & .01 & .09 \\
OE & -.07 & & \\
\hline
\end{tabular}

Note: ${ }^{\$} P<.10,{ }^{*} P<.05,{ }^{* *} P<.01$. Baseline: resting RSA at baseline; suppression: change in RSA (baseline minus paced arithmetic); recovery: change in RSA (relaxation minus paced arithmetic); MC: Marlowe-Crowne; BAS: Behavioral Activation Scale; BIS: Behavioral Inhibition Scale; Anxietysocial: social anxiety subscale from Self-Consciousness Scale; Anxiety Manifest: Taylor Manifest Anxiety Scale; OE: openness to experience. 


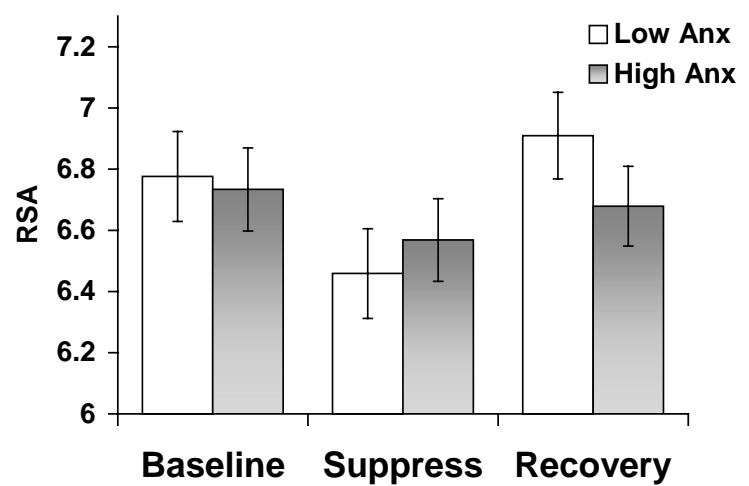

Fig. 1. Cardiac Vagal Tone (indexed by RSA) at baseline, suppression, and recovery phases for high and low socially-anxious individuals.

Anxiety and Social Anxiety scales, was partially supported. Zero-order correlations (see Table 2) revealed that although baseline vagal tone was unrelated to either anxiety measure, modulation of vagal tone in response to the suppression and recovery tasks was related to social anxiety $(r=-.22$ and $r=-.33, P<.05$ and $P<.01$, respectively) but not manifest anxiety. Repeated-measures ANOVAs using median splits to define highand low-anxious subjects essentially corroborated the correlational findings. For social anxiety, the main effect of task was qualified by a nearly significant interaction (see Fig. 1) with anxiety $(F(2,188)=2.8, P<.07)$. Follow-up repeated-measures ANOVAs for high- and low-anxiety subjects separately were conducted based on the hypothesis that high-anxious subjects would show poor modulation; as predicted, low socially-anxious subjects modulated vagal tone in response to task $(F(2,88)=9.0, P<.001)$ but high socially anxious subjects did not significantly modulate in response to task $(F(2,104)=$ $1.5, P>.23)$. For manifest anxiety, no effects involving anxiety emerged.

\subsubsection{Defensiveness}

It was hypothesized that subjects exhibiting lower baseline vagal tone and less suppression of it in response to a stressful task would be more defensive, as measured by the Marlowe-Crowne short form. Although the correlations in Table 2 did not support this hypothesis, the repeated-measures ANOVA partially supported the prediction: highly defensive individuals did exhibit lower resting vagal tone, but did not exhibit less capacity for suppression. A main effect of task and a main effect of defensiveness $(F(1,96)=6.3$, $P<.05$ ) emerged in the absence of a significant interaction (see Fig. 2). Thus, high defensive subjects exhibited lower vagal tone across all tasks, but appeared equally capable of modulating RSA in response to the paced serial arithmetic task and recovery phase.

\subsubsection{Behavioral activation and inhibition}

Support for the relationship between vagal tone and behavioral activation was found for baseline level as predicted (see Table 2). Subjects with higher resting vagal tone had higher BAS scores, and in particular higher scores on the reward responsiveness subscale. The 


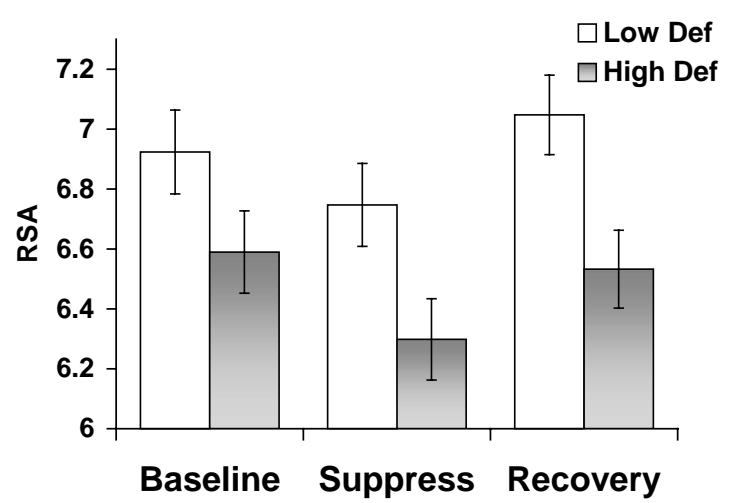

Fig. 2. Cardiac Vagal Tone (indexed by RSA) at baseline, suppression, and recovery phases for high and low defensive individuals.

repeated measures ANOVA with subjects split at the median on BAS score replicated these findings. A main effect of task and a main effect of BAS Score $(F(1,94)=6.1, P<.05)$ emerged in the absence of a significant interaction. Subjects with high BAS scores exhibited significantly higher vagal tone than low BAS subjects, with no differential modulation in response to tasks.

No support was found in the correlational analyses for the hypotheses that higher vagal tone would predict lower behavioral inhibition. The repeated measures ANOVA failed to find a main effect of BIS Score $(F(1,90)=0.30$, ns), nor of Gender $(F(1,90)=0.31$, $\mathrm{ns})$, but did reveal a significant BIS-level by Gender interaction $(F(1,90)=4.5, P<.05)$. Whereas women with high BIS scores did not differ in overall vagal tone $(6.50 \pm 0.14)$ from their low BIS counterparts $(6.8 \pm 0.19)$, men with high BIS scores displayed marginally $(P<.08)$ higher overall vagal tone $(7.0 \pm 0.22)$ than their low BIS counterparts $(6.6 \pm 0.18)$.

\subsubsection{Intercorrelation of personality measures}

Table 3 presents the intercorrelation of the self-report measures. Consistent with expectations, higher defensiveness as assessed by the Marlowe-Crowne portended lower social and

Table 3

Correlations Among Self-report and Self-Guide Measures

\begin{tabular}{|c|c|c|c|c|c|c|c|c|}
\hline & MC & BAS $_{\text {Total }}$ & $\mathrm{BAS}_{\mathrm{Fun}}$ & BAS $_{\text {Reward }}$ & BAS $_{\text {Drive }}$ & BIS & AnxietySocial & Anxiety Manifest \\
\hline BAS $_{\text {Total }}$ & -.08 & & & & & & & \\
\hline BAS $_{\text {Fun }}$ & -.13 & $.68^{* *}$ & & & & & & \\
\hline BAS $_{\text {Reward }}$ & -.05 & $.70^{* *}$ & $.30^{* *}$ & & & & & \\
\hline BAS $_{\text {Drive }}$ & -.08 & $.83^{* *}$ & $.34^{* *}$ & $.35^{* *}$ & & & & \\
\hline BIS & -.19 & .03 & $-.23^{*}$ & $.38^{* *}$ & -.05 & & & \\
\hline Anxiety & $-.21^{*}$ & $-.22^{*}$ & -.18 & -.02 & $-.27^{* *}$ & $.33^{* *}$ & & \\
\hline Anxiety Manifest & $-.29^{* *}$ & .05 & -.08 & $.23^{*}$ & .00 & $.55^{* *}$ & $.45^{* *}$ & \\
\hline $\mathrm{OE}$ & -.06 & .14 & $.22^{*}$ & .18 & -.01 & -.07 & -.11 & -.09 \\
\hline
\end{tabular}

Note: $* P<.05 ; * * P<.01$. MC: Marlowe-Crowne; BAS: Behavioral Activation Scale; BIS: Behavioral Inhibition Scale; Anxietysocial: social anxiety subscale from Self-Consciousness Scale; Anxiety Manifest: Taylor Manifest Anxiety Scale; OE: openness to experience. 
general anxiety scores. Higher behavioral activation predicted lower social anxiety, but not general anxiety. Higher behavioral inhibition predicted higher social and general anxiety. Higher Openness to Experience predicted greater Fun Seeking (BAS subscale).

\section{Discussion}

The present data provided modest support for the hypotheses. Results revealed that subjects who showed less suppression of vagal tone during a stressful task were higher in social anxiety than subjects who showed robust suppression. Second, low-defensive subjects exhibited higher vagal tone across all tasks. Third, subjects with greater behavioral activation, particularly with respect to potential rewards, exhibited greater vagal tone. Finally, behavioral inhibition scores interacted with gender, but no unequivocal decomposition of the interaction emerged. Men higher in behavioral inhibition had nonsignicantly higher vagal tone, whereas no differences whatsoever were shown for low versus high-BIS women.

\subsection{Vagal tone and anxiety}

Contrary to findings with clinically anxious populations (Lyonfields et al., 1995; Friedman and Thayer, 1998), vagal tone as indexed by RSA was not found to relate to scores on self-reported manifest anxiety in this non-clinical population. Higher social anxiety was, however, associated with less suppression of vagal tone during a demanding task and less recovery toward baseline levels following the task. This may be due to the nature of the experimental situation, which in this case involved interacting with a young adult experimenter and having to perform a stressful task in response to paced demands by that experimenter. Because subjects scoring higher in a more global measure of anxiety did not show this failure to suppress vagal tone, however, it may be that social anxiety taps a kind of self-conscious worry (Fenigstein, 1975), whereas manifest anxiety taps a general tendency toward agitation that may not have been salient in this experimental context. It may also be the case that the relationship between manifest anxiety and vagal tone is only evident at clinically significant levels of anxiety (e.g. as those in Lyonfields et al., 1995; Friedman and Thayer, 1998).

\subsection{Vagal tone and defensiveness}

Defensive subjects - those who tended to deny common frailties and endorse uncommon virtues - exhibited significantly lower vagal tone across all three task conditions than did their less defensive counterparts. Defensive subjects did not differ, however, in their ability to suppress vagal influence in response to a demanding task. To the degree that higher resting vagal tone is thought to promote the capacity to attend to stimuli, this finding would seem to be partially consistent with the observation that defensive subjects, found here to have diminished resting vagal tone, are less able or willing to attend to threatening stimuli (e.g., Weinberger et al., 1979). More work remains to be done in this area, however, as one might expect lower resting vagal influence to attenuate attention to stimuli without respect to stimulus valence; yet high defensive subjects appear to demonstrate a valence 
specific attenional bias. Future research might profitably examine vagal suppression in high-defensive subjects as they are confronted with a more personally threatening task demand, such as lewd words (Kline et al., 1998a,b) or previously self-ascribed negative traits.

The fact that a relationship between vagal tone and defensiveness emerged using a median split but not at the level of a zero-order correlation with baseline RSA (see Table 2) is puzzling. The main effect in the median split analysis, however, reflected a relationship across three vagal tone assessment periods (baseline, arithmetic task, recovery phase). To test whether a similar correlational relationship would be observed, the mean vagal tone (as indexed by RSA) across all three task conditions was found to correlate -.17 ( $p=.05$, one-tailed) with Marlowe-Crowne score. This overall correlation suggests that the result identified using the median split approach is also apparent, although relatively small, using a correlational approach. Additional data argue against the possibility that the present finding is an isolated chance finding. Dikman et al. (2001), in an analysis of the subjects reported in Dikman and Allen (2000), found that the correlation between RSA and defensiveness was significant $(r=-.41, P<.05)$ in a sample of 34 college students. This relationship was not moderated by gender, nor by participant's socialization status as defined by the socialization scale of the California Psychological Inventory (Gough, 1994).

\subsection{Vagal tone and behavioral activation/inhibition}

\subsubsection{Behavioral activation}

For both men and women, higher BAS scores-and in particular higher scores on the reward responsiveness subscale-were predictive of higher resting vagal tone as measured by baseline RSA. This finding is generally consistent with the finding of greater behavioral and emotional reactivity in individuals with higher vagal tone (Eisenberg et al., 1995) but at first inspection it is puzzling in that vagal suppression was uncorrelated to BAS. These findings are consistent, however, with Beauchaine's (2001) model that low baseline vagal tone is related to negative emotional traits and that high vagal withdrawal is related to negative emotional states. Those high in BAS have been hypothesized to be less likely to experience trait emotional negativity and psychopathology (Depue and Iacono, 1989; Fowles, 1988; Harmon-Jones and Allen, 1997), and it is, therefore, consistent that they demonstrate high vagal tone as indexed by RSA. Thus although those high in BAS sensitivity appear to have potentially a greater capacity to attend and orient, the present task may not have been optimal to differentiate the extent to which high and low BAS subjects would engage and, therefore, modulate vagal tone. Instead of the use of a stressful task, future research might investigate the use of a reward incentive, which should differentially engage high and low BAS individuals.

\subsubsection{Behavioral inhibition}

The present data generally suggest that that BIS scores and vagal tone are unrelated, although the interaction with gender identified a nonsignificant trend of higher vagal tone among men with higher BIS scores. In the absence of previous findngs or a strong theoretical prediction with respect to vagal tone and BIS, this finding is reported but not interpreted further pending potential replication. 


\subsection{Limitations of the current study}

Two limitations of the current study must be acknowledged. First, no concurrent respiratory data were collected to assess the impact of respiration frequency and amplitude, although measures that utilize respiration in the calculation of an estimate of vagal tone correlate quite highly with a measure of RSA like that in the present study (Grossman et al., 1990). Further, because the baseline measure of vagal tone in the present study was conducted under resting conditions and after subjects had been at rest for 5-10 min (during the hookup of electrodes), there is no strong reason to believe that subjects' breathing patterns were atypical in this study. Second, although those subjects with highly deviant RSA values were excluded, subjects were not asked to disclose respiratory or cardiovascular conditions, nor was family history examined to rule out subjects who had notable histories for hypertension or other cardiovascular disorder.

\subsection{Summary}

Cardiac vagal tone has been shown in previous studies to predict behavioral and emotional tendencies across infant, child, and adult populations. Studies have generally suggested that individuals who have greater vagal influence are quicker to respond to stimuli, respond more strongly, calm down again more quickly, and are more emotionally expressive than individuals whose vagal tone and capacity for vagal suppression is lower. The present study adds to this literature by linking vagal tone to more commonly used motivation-related measures of personality, finding that subjects high in approach motivation and low in defensiveness show higher vagal tone as measured by RSA, and that those lower in social anxiety show better modulation of vagal tone in response to challenge. Future studies might expand this work by examining vagal tone as a predictor of other self-report or interview measures that tap personality, coping style, attentional performance, and relational and communication patterns. Future studies might also examine vagal tone as a predictor of behavior in laboratory based social exchanges, or in tasks varying in incentive structure (e.g., motivational rewards and punishments). Finally, given that symptom change in depression appears to covary with enhanced vagal tone (Chambers and Allen, 2002), it would be worth investigating whether interventions can induce either temporary or lasting change in vagal tone, and whether such change is associated with clinically significant changes in defensiveness, anxiety, and other motivation-related personality characteristics.

\section{Acknowledgements}

The authors wish to thank Kelli Glass and Lauren Stern for assistance in data collection and entry, and Jim Coan for helpful comments on an earlier draft of this mansucript. We are indebted to Steve Porges for generously providing us with the MX Edit software and manual.

\section{References}

Allen, J.J.B., 2002. Calculating metrics of cardiac chronotropy: a pragmatic overview. Psychophysiology 39, S18. 
Beauchaine, T., 2001. Vagal tone, development, and Gray's motivational theory: toward an integrated model of autonomic nervous system functioning in psychopathology. Development and Psychopathology 13, 183-214.

Berntson, G.G., Bigger, J.T., Eckberg, D.L., Grossman, P., Kaufmann, P.G., Malik, M., Nagaraja, H.N., Porges, S.W., Saul, J.P., Stone, P.H., Van Der Molen, M.W., 1997. Heart rate variability: origins, methods, and interpretative caveats. Psychophysiology 34, 623-648.

Berntson, G.G., Cacioppo, J.T., Quigley, K.S., 1993. Respiratory sinus arrhythmia: autonomic origins, physiological mechanisms, and psychophysiological implications. Psychophysiology 30, 183-196.

Carney, R.M., Rich, M.W., TeVelde, A., Saini, J., et al., 1995. The relationship between heart rate, heart rate variability and depression in patients with coronary artery disease. Journal of Psychosomatic Research 32, $159-164$.

Carver, C.S., White, T.L., 1994. Behavioral inhibition, behavioral activation, and affective responses to impending reward and punishment: the BIS/BAS scales. Journal of Personality \& Social Psychology 67 (2), 319-333.

Chambers, A.S., Allen, J.J.B., 2002. Vagal tone as indicator of treatment response in major depression. Psychophysiology 39, 861-864.

Coan, J.A., Allen, J.J.B., 2003. The state and trait nature of frontal EEG asymmetry in emotion. In: Hugdahl, K., Davidson, R.J. (Eds.), The Asymmetrical Brain. MIT Press, Cambridge, MA, pp.565-615.

Coan, J.A., Allen, J.J.B., 2004. Frontal EEG asymmetry as a moderator and mediator of emotion. Biological Psychology 67, 7-50.

Cole, P.M., Zahn-Waxler, C., Fox, N.A., Usher, B.A., Welsh, J.D., 1996. Individual differences in emotion regulation and behavior problems in school children. Journal of Abnormal Psychology 105, 518-529.

Cook 3rd, E.W., Miller, G.A., 1992. Digital filtering: background and tutorial for psychophysiologists [erratum appears in Psychophysiology 1992 Jul;29(4)470]. Psychophysiology 29 (3), 350-367.

Costa, P.T., McCrae, R.R., 1985. The NEO Personality Inventory Manual. Psychological Assessment Resources, Odessa, FL.

Costa, P.T., McCrae, R.R., 1992. Four ways five factors are basic. Personality and Individual Differences 13, 653-665.

Davidson, R.J., 1998. Affective style and affective disorders: perspectives from affective neuroscience. Cognition \& Emotion 12, 307-330.

Depue, R.A., Iacono, W.G., 1989. Neurobehavioral aspects of affective disorders. Annual Review of Psychology 40, 457-492.

Dikman, Z.V., Allen, J.J.B., 2000. Error monitoring during reward and avoidance learning in high and low-socialized individuals. Psychophysiology 37, 43-54.

Dikman, Z.V., Allen, J.J.B., Movius, H.L., 2001. Vagal tone and defensiveness: more data to make defensive people defensive. Psychophysiology 38, S36.

Eisenberg, N., Fabes, R.A., Murphy, B., Maszk, P., Smith, M., Karbon, M., 1995. The role of emotionality and regulation in children's social functioning: a longitudinal study. Child Development 66, 1360-1384.

Fabes, R.A., Eisenberg, N., 1997. Regulatory control and adults' stress-related responses to daily life events. Journal of Personality \& Social Psychology 73, 1107-1117.

Fenigstein, A., Scheier, M.F., Buss, A.H., 1975. Public and private self-consciousness: assessment and theory. Journal of Consulting and Clinical Psychology 36, 1241-1250.

Field, T., Woodson, R., Greenberg, R., Cohen, D., 1982. Discrimination and imitation of facial expressions by neonates. Science 218, 179-181.

Fowles, D.C., 1987. Application of a behavioral theory of motivation to the concepts of anxiety and impulsivity. Journal of Research in Personality 21, 417-435.

Fowles, D.C., 1988. Psychophysiology and psychopathology: a motivational approach. Psychophysiology 25, 373-391.

Fowles, D.C., 1994. A motivational theory of psychopathology. Nebraska Symposium on Motivation 41, 180-238.

Fox, N.A., 1989. Psychophysiological correlates of emotional reactivity during the first year of life. Development Psychology 25, 364-372.

Friedman, B.H., Thayer, J.F., 1998. Autonomic balance revisited: panic anxiety and heart rate variability. Journal of Psychosomatic Research 44, 133-151.

George, D.T., Nutt, D.J., Walker, W.V., Porges, S.W., Adinoff, B., Linnoila, M., 1989. Lactate and hyperventilation substantially attenuate vagal tone in normal volunteers. A possible mechanism of panic provocation. Archives of General Psychiatry 46 (2), 153-156. 
Gottman, J.M., Katz, L.F., Hooven, C., 1996. Parental meta-emotion philosophy and the emotional life of families: theoretical models and preliminary data. Journal of Family Psychology 10, 243-268.

Gough, H.G., 1994. Theory, development, and interpretation of the CPI Socialization scale. Psychological Reports $75,651-700$.

Grossman, P., van Beek, J., Wientjes, C., 1990. A comparison of three quantification methods for estimation of respiratory sinus arrhythmia. Psychophysiology 27, 702-714.

Harmon-Jones, E., Allen, J.J.B., 1997. Behavioral activation sensitivity and resting frontal EEG asymmetry: covariation of putative indicators related to risk for mood disorders. Journal of Abnormal Psychology 106, $159-163$.

Harmon-Jones, E., Allen, J.J.B., 1998. Anger and frontal brain activity: EEG asymmetry consistent with approach motivation despite negative affective valence. Journal of Personality \& Social Psychology 74, 1310-1316.

Hughes, J.W., Stoney, C.M., 2000. Depressed mood is related to high-frequency heart rate variability during stressors. Psychosomatic Medicine 62, 796-801.

Katz, L.F., Gottman, J.M., 1995. Vagal tone protects children from marital conflict. Development and Psychopathology 7, 83-92.

Katz, L.F., Gottman, J.M., 1997. Buffering children from marital conflict and dissolution. Journal of Consulting and Clinical Psychology 26, 157-171.

Lane, R.D., Merikangas, K.R., Schwartz, G.E., Huang, S.S., et al., 1990. Inverse relationship between defensiveness and lifetime prevalence of psychiatric disorder. American Journal of Psychiatry 147, 573-578.

Kline, J.P., Schwartz, G.E., Allen, J.J.B., Dikman, Z.V., 1998a. Perceptual and electroencephalographic registration of masked emotional words in defensiveness: an exploratory study. Personality and Individual Differences 24, 499-512.

Kline, J.P., Allen, J.J.B., Schwartz, G.E., 1998b. Is left frontal brain activation in defensiveness gender specific? Journal of Abnormal Psychology 107, 149-153.

Lyonfields, J.D., Borkovec, T.D., Thayer, J.F., 1995. Vagal tone in Generalized Anxiety Disorder and the effects of aversive imagery and worrisome thinking. Behavior Therapy 26, 457-466.

McCrae, R.R., 1996. Social consequences of experiential openness. Psychological Bulletin 120, 323-337.

McCrae, R.R., Costa, P.T., 1994. The stability of personality: observations and evaluations. Current Directions in Psychological Science 3, 173-175.

McCrae, R.R., John, O.P., 1992. An introduction to the five-factor model and its applications. Journal of Personality $60,175-215$.

Miller, S.B., 1993. Cardiovascular reactivity in anger-defensive individuals: the influence of task demands. Psychosomatic Medicine 55, 79-85.

Moser, M., Lehofer, M., Hoehn-Saric, R., McLeod, D.R., Hildebrandt, G., Steinbrenner, B., et al., 1998. Increased heart rate in depressed subjects in spite of unchanged autonomic balance? Journal of Affective Disorders 48 (2-3), 115-124.

Papillo, J.F., Shapiro, D., 1990. The cardiovascular system. In: Cacioppo, J.T., Tassinary, L.G. (Eds.), Principles of psychophysiology: Physical, Social, and Inferential Elements. Cambridge University Press, Cambridge, pp. 456-512.

Pine, D.S., Wasserman, G.A., Miller, L., Coplan, J.D., Bagiella, E., Kovelenku, P., Myers, M.M., Sloan, R.P., 1998. Heart period variability and psychopathology in urban boys at risk for delinquency. Psychophysiology $35,521-529$.

Porges, S.W., 1991. Vagal tone: an autonomic mediator of affect. In: Garber, J., Dodge, K.A. (Eds.), The Development of Emotion Regulation and Dysregulation. Cambridge University Press, Cambridge, pp. 111-128.

Porges, S.W., 1995. Orienting in a defensive world: Mammalian modifications of our evolutionary heritage. A polyvagal theory. Psychophysiology 32, 301-318.

Porges, S.W., Arnold, W.R., Forbes, E.J., 1973. Heart rate variability: an index of attentional responsivity in newborns. Developmental Psychology 8, 85-92.

Porges, S.W., Doussard-Roosevelt, J.A., Maiti, A.K., 1994. Vagal tone and the physiological regulation of emotion. Monographs of the Society for Research in Child Development 59, 167-186.

Porges, S.W., Doussard-Roosevelt, J.A., Portales, A.L., Greenspan, S.I., 1996. Infant regulation of the vagal "brake" predicts child behavior problems: a psychobiological model of social behavior. Developmental Psychobiology $29,697-712$. 
Porges, S.W., Walter, G.F., Korb, R.J., Sprague, R.L., 1975. The influence of methylphenidate on heart rate and behavioral measures of attention in hyperactive children. Child Development 46, 727-733.

Rechlin, T., Weis, M., Spitzer, A., Kaschka, W.P., 1994. Are affective disorders associated with alterations of heart rate variability. Journal of Affective Disorders 32 (4), 271-275.

Rechlin, T., Weis, M., Kaschka, W.P., 1995. Is diurnal variation of mood associated with parasympathetic activity? Journal of Affective Disorders 34 (3), 249-255.

Reynolds, W.M., 1982. Development of reliable and valid short forms of the Marlowe-Crowne Social Desirability Scale. Journal of Clinical Psychology 38, 119-125.

Richards, J.E., 1985. Respiratory sinus arrhythmia predicts heart rate and visual responses during visual attention in 14- and 20-week-old infants. Psychophysiology 22, 101-109.

Roose, S.P., Glassman, A.H., Dalack, G.W., 1989. Depression, heart disease, and tricyclic antidepressants. Journal of Clinical Psychiatry 50, 12-16.

Schwartz, G.E., 1990. Psychobiology of systems and health: a systems approach. In: Singer, J. (Ed.), Repression and dissociation. University of Chicago Press, Chicago.

Shapiro, D., Goldstein, I.B., Jammer, L.D., 1995. Effects of anger/hostility, defensiveness, gender, and family history of hypertension on cardiovascular reactivity. Psychophysiology 32 (5), 425-435.

Sloan, R.P., Bigger Jr., J.T., 1991. Biobehavioral factors in Cardiac Arrhythmia Pilot Study (CAPS). Review and examination. Circulation 83 (4 Suppl), II52-II57.

Sloan, R.P., Korten, J.B., Myers, M.M., 1991. Components of heart rate reactivity during mental arithmetic with and without speaking. Physiology \& Behavior 50, 1039-1045.

Stifter, C.A., Fox, N.A., Porges, S.W., 1989. Facial expressivity and vagal tone in 5- and 10-month-old infants. Infant Behavior and Development 12, 127-137.

Taylor, J.A., 1953. A personality scale of manifest anxiety. Journal of Abnormal and Social Psychology 48, 285-290.

Weinberger, D.A., 1990. The construct validity of the repressive coping style. In: Singer, J. (Ed.), Repression and Dissociation. University of Chicago Press, Chicago.

Weinberger, D.A., Schwartz, G.E., Davidson, R.J., 1979. Low-anxious, high-anxious and repressive coping styles: Psychometric patterns and behavioral and physiological responses to stress. Journal of Abnormal Psychology 88, 369-380. 